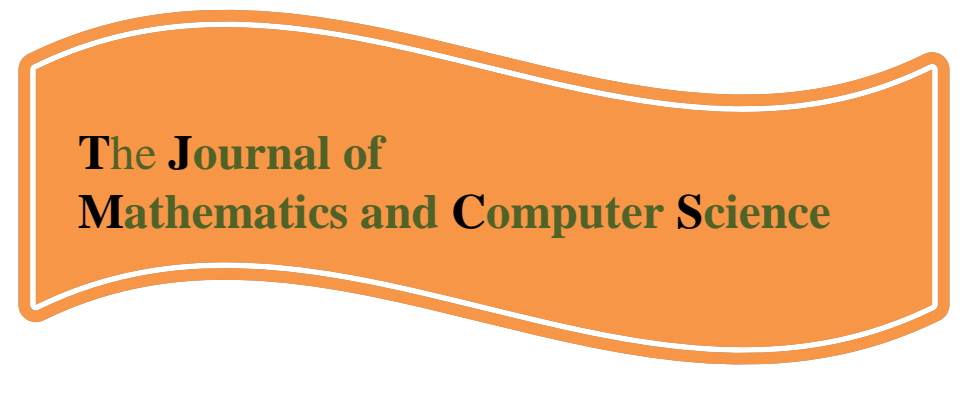

Available online at

http://www.TIMCS.com

The Journal of Mathematics and Computer Science Vol .5 No.1 (2012) 40-52

\title{
APPLICATION OF HOMOTOPY PERTURBATION TRANSFORM METHOD TO LINEAR AND NON-LINEAR SPACE-TIME FRACTIONAL REACTION- DIFFUSION EQUATIONS
}

\author{
Jagdev Singh \\ Department of Mathematics, Jagan Nath University, Jaipur, Rajasthan, India \\ E-mail: jagdevsinghrathore@gmail.com \\ Devendra Kumar \\ Department of Mathematics, Jagan Nath Gupta Inst. of Engg. \& Tech., Jaipur, Rajasthan, India \\ E-mail: devendra.maths@gmail.com

\section{Sushila} \\ Department of Physics, Jagan Nath University, Jaipur, Rajasthan, India \\ E-mail: sushila.jag@gmail.com

\section{Sumit Gupta} \\ Department of Mathematics, Jagan Nath Gupta Inst. of Engg. \& Tech., Jaipur, Rajasthan, India \\ E-mail: guptasumit.edu@gmail.com
}

Received: November 2011, Revised: December 2012

Online Publication: December 2012

\begin{abstract}
In this paper, we obtain the analytical solutions of linear and non-linear space-time fractional reaction-diffusion equations on a finite domain by the application of homotopy perturbation transform method (HPTM). The HPTM is a combined form of the Laplace transform method with the homotopy perturbation method. Some examples are also given. Numerical results show that the HPTM is easy to implement and accurate when applied to linear and non-linear space-time fractional reaction-diffusion equations.
\end{abstract}

Keywords: Homotopy perturbation transform method, Laplace transform, fractional reactiondiffusion equation, Caputo time-fractional derivative, Caputo space-fractional derivative.

2010 Mathematics Subject Classification: 35R11, 44A10, 35C10. 


\section{Introduction}

In recent years, it has turned out that many phenomena in engineering, physics, chemistry and other sciences can be described very successfully by models using mathematical tools from fractional calculus. For example, the nonlinear oscillation of earthquake can be modeled with fractional derivatives and the fluid-dynamic traffic model with fractional derivatives can eliminate the deficiency arising from the assumption of continuum traffic flow. Fractional derivatives are also used in modeling of many chemical processes, mathematical biology and many other problems in physics and engineering. These findings invoked the growing interest of studies of the fractional calculus in various fields such as physics, chemistry and engineering.

Fractional differential equations have gained importance and popularity during the past three decades or so, mainly due to exact description of nonlinear phenomena, especially in fluid mechanics, e.g. nano-hydrodynamics, where continuum assumption does not well, and fractional model can be considered to be a best candidate. Hence, great attention has been given to finding solutions of fractional differential equations. Most fractional differential equations do not have exact analytical solutions, therefore approximate and numerical techniques must be used. Variational iteration method (VIM) [16] was first proposed to solve fractional differential equations with greatest success. Many authors found VIM as an effective way to solving linear and non-linear fractional differential equations [7,33]. The VIM was also used by many authors to study the various physical problems $[23,26,36]$. The homotopy perturbation method (HPM) was first introduced by J.H. He [17]. The HPM was applied to solve the $12^{\text {th }}$ order boundary value problems [35]. In recent years Momani and Odibat [29], Ganji et al. [9], Yildirim [42-44], Yildirim and Sezer [45] and Jafari and Momani [20] applied the HPM to fractional differential equations and revealed that HPM is an alternative analytical method for solving fractional differential equations. Momani et al. [30] and Odibat and Momani [34] compared solutions procedure between VIM and HPM.

In this paper, we use the homotopy perturbation transform method (HPTM) [22] for solving linear and non-linear space-time fractional reaction-diffusion equations on a finite domain. It is worth mentioning that this method is an elegant combination of the Laplace transformation, the HPM and He's polynomials and is mainly due to Ghorbani [10,11]. The use of He's polynomials in the nonlinear term was first introduced by Ghorbani $[10,11]$. This algorithm provides the solution in a rapid convergent series which may lead to the solution in a closed form. The advantage of this method is its capability of combining two powerful methods for obtaining exact solutions for nonlinear equations.

In recent years, fractional reaction-diffusion models are studied due to their usefulness and importance in many areas of science and engineering. The reaction-diffusion equations arise naturally as description models of many evolution problems in the real world, as in chemistry $[39,40]$, biology [32], etc. As is well known, complex behavior is peculiarity of systems modeled by reaction diffusion equations and the Belousov-Zhabotinskii reaction $[31,41]$ provides a classic example. The reaction-diffusion equations describe a population of diploid individuals (i.e., the ones that carry two genes) distributed in a two-dimensional habitat. Assuming that a gene occurs in two forms $a$ and $A$, called alleles, one can divide the population into three genotypes $a a, a A$ and $A A$. The reaction-diffusion equations are employed to describe the co-oxidation on Pt (110) [2], the study of temporal and spatial patterns of cytoplasmic $\mathrm{Ca}^{+2}$ dynamics under the effects of $\mathrm{Ca}^{+2}$-release activated $\mathrm{Ca}^{+2}$ (CRAC) channels in T cells [6], problems in finance [12,25,37] and hydrology [3]. Burke et al. [4] obtained solutions for an enzyme-suicide substrate reaction with an instantaneous point source of substrate. In 1993, Grimson and Barker [15] introduced a continuum model for the spatio-temporal growth of bacterial colonies on the surface of a solid substrate which utilizes a reaction-diffusion equation for growth. Many cellular and sub-cellular biological processes [8] can be described in terms of diffusing and chemically reacting species (e.g. enzymes). A traditional 
approach to the mathematical modeling of such reaction-diffusion processes is to describe each biochemical species by its (spatially dependent) concentration. In recent time, interest in fractional reaction-diffusion equations $[1,13,14,18,21,38,45]$ has increased because the equation exhibits selforganization phenomena and introduces a new parameter, the fractional index, into the equation. Additionally, the analysis of fractional reaction-diffusion equations is of great importance from the analytical and numerical point of view.

The Riemann-Liouville fractional integration of order $\alpha$ is defined as [27]

$$
J_{a}^{\alpha} f(x, t)=\frac{1}{\Gamma(\alpha)} \int_{a}^{x}(x-u)^{\alpha-1} f(u, t) d u, x>0 .
$$

The following fractional derivative of order $\alpha>0$ is introduced by Caputo [5]; see also Kilbas et al. [24] in the form

$$
\begin{aligned}
{ }_{0} \mathrm{D}_{\mathrm{t}}^{\alpha} \mathrm{f}(\mathrm{x}, \mathrm{t}) & =\frac{1}{\Gamma(\mathrm{m}-\alpha)} \int_{0}^{\mathrm{t}} \frac{\mathrm{f}^{(\mathrm{m})}(\mathrm{x}, \tau) \mathrm{d} \tau}{(\mathrm{t}-\tau)^{\alpha+1-\mathrm{m}}}, \mathrm{m}-1<\alpha \leq \mathrm{m}, \operatorname{Re}(\alpha)>0, \mathrm{~m} \in \mathrm{N}, \\
& =\frac{\partial^{\mathrm{m}} \mathrm{f}(\mathrm{x}, \mathrm{t})}{\partial \mathrm{t}^{\mathrm{m}}}, \quad \text { if } \alpha=\mathrm{m},
\end{aligned}
$$

where $\frac{\partial^{m} f(x, t)}{\partial t^{m}}$ is the $m$-th partial derivative of $f(x, t)$ with respect to $t$.

The Laplace transform of the Caputo derivative is given by Caputo [5]; see also Kilbas et al. [24] in the form

$$
\mathrm{L}\left\{{ }_{0} \mathrm{D}_{\mathrm{t}}^{\alpha} \mathrm{f}(\mathrm{x}, \mathrm{t})\right\}=\mathrm{s}^{\alpha} L[\mathrm{f}(\mathrm{x}, \mathrm{t})]-\sum_{\mathrm{r}=0}^{m-1} \mathrm{~s}^{\alpha-\mathrm{r}-1} \mathrm{f}^{(\mathrm{r})}(\mathrm{x}, 0+),(\mathrm{m}-1<\alpha \leq \mathrm{m}) .
$$

The Liouville fractional derivative of order $\alpha$ is defined in [24, Section 24.2] in the form

$$
\frac{\partial^{\alpha}}{\partial \mathrm{x}^{\alpha}} \mathrm{f}(\mathrm{x}, \mathrm{t})=\frac{1}{\Gamma(\mathrm{m}-\alpha)}\left(\frac{\partial}{\partial \mathrm{x}}\right)^{\mathrm{m}} \int_{-\infty}^{\mathrm{x}} \frac{\mathrm{f}(\mathrm{y}, \mathrm{t})}{(\mathrm{x}-\mathrm{y})^{\alpha-\mathrm{m}+1}} \mathrm{dy}, \quad(\mathrm{x} \in \mathrm{R}, \alpha>0, \mathrm{~m}=[\alpha]+1)
$$

where $[\alpha]$ is the integral part of $\alpha, \alpha>0$.

\section{HPTM solutions of linear space-time fractional reaction-diffusion equation}

In this paper, we first consider the linear space-time fractional reaction-diffusion equation of the form:

$$
\begin{aligned}
& D_{t}^{\alpha} u(x, t)=b(x) D_{x}^{\beta} u(x, t)-c(x) u(x, t)+f(x, t), 0<x<L, t>0,0<\alpha \leq 1,1<\beta \leq 2, \\
& u(x, 0)=p(x), \\
& u(0, t)=q_{1}(t), \\
& u_{x}(0, t)=q_{2}(t) .
\end{aligned}
$$

Taking the Laplace transform on both sides of Eq. (5) and using (6), we get

$$
L[u(x, t)]=\frac{p(x)}{s}+\frac{1}{s^{\alpha}} b(x) L\left[D_{x}^{\beta} u(x, t)\right]-\frac{1}{s^{\alpha}} c(x) L[u(x, t)]+\frac{1}{s^{\alpha}} L[f(x, t)] .
$$

Applying the inverse Laplace transform on both sides of Eq. (9), we get

$$
u(x, t)=p(x)+L^{-1}\left[\frac{b(x)}{s^{\alpha}} L\left[D_{x}^{\beta} u(x, t)\right]-\frac{c(x)}{s^{\alpha}} L[u(x, t)]\right]+J_{t}^{\alpha} f(x, t) .
$$

Now, we apply the homotopy perturbation method

$$
u(x, t)=\sum_{n=0}^{\infty} p^{n} u_{n}(x, t) .
$$

Substituting Eq. (11) in Eq. (10), we get 


$$
\begin{aligned}
& \sum_{n=0}^{\infty} p^{n} u_{n}(x, t)= \\
& \quad p(x)+p\left(L^{-1}\left[\frac{1}{s^{\alpha}} b(x) L\left[D_{x}^{\beta} \sum_{n=0}^{\infty} p^{n} u_{n}(x, t)\right]-\frac{c(x)}{s^{\alpha}} L\left[\sum_{n=0}^{\infty} p^{n} u_{n}(x, t)\right]\right]\right)+J_{t}^{\alpha} f(x, t) .
\end{aligned}
$$

Comparing the coefficients of the like terms of $p$, we have

$$
\begin{aligned}
p^{0}: u_{0}(x, t) & =p(x)+J_{t}^{\alpha} f(x, t), \\
p^{1}: u_{1}(x, t) & =L^{-1}\left[\frac{b(x)}{s^{\alpha}} L\left[D_{x}^{\beta} u_{0}(x, t)\right]-\frac{c(x)}{s^{\alpha}} L\left[u_{0}(x, t)\right]\right] \\
= & {\left[b(x) D_{x}^{\beta}-c(x)\right]\left[p(x) \frac{t^{\alpha}}{\Gamma(\alpha+1)}+J_{t}^{2 \alpha} f(x, t)\right] . }
\end{aligned}
$$

Proceeding in a similar manner, we get

$$
\begin{aligned}
& p^{2}: u_{2}(x, t)=\left[b(x) D_{x}^{\beta}-c(x)\right]^{2}\left[p(x) \frac{t^{2 \alpha}}{\Gamma(2 \alpha+1)}+J_{t}^{3 \alpha} f(x, t)\right], \\
& p^{3}: u_{3}(x, t)=\left[b(x) D_{x}^{\beta}-c(x)\right]^{3}\left[p(x) \frac{t^{3 \alpha}}{\Gamma(3 \alpha+1)}+J_{t}^{4 \alpha} f(x, t)\right], \\
& \vdots \\
& p^{n}: u_{n}(x, t)=\left[b(x) D_{x}^{\beta}-c(x)\right]^{n}\left[p(x) \frac{t^{n \alpha}}{\Gamma(n \alpha+1)}+J_{t}^{(n+1) \alpha} f(x, t)\right],
\end{aligned}
$$

and so on; in this manner, the rest of components of the homotopy perturbation series can be obtained. Thus, we have the solution in series form is given by

$$
\begin{aligned}
\mathrm{u}(\mathrm{x}, \mathrm{t}) & =\mathrm{p}(\mathrm{x})+\mathrm{J}_{\mathrm{t}}^{\alpha} f(x, t)+\left[b(x) D_{x}^{\beta}-c(x)\right]\left[p(x) \frac{t^{\alpha}}{\Gamma(\alpha+1)}+J_{t}^{2 \alpha} f(x, t)\right]+\cdots \\
& +\left[b(x) D_{x}^{\beta}-c(x)\right]^{n}\left[p(x) \frac{t^{n \alpha}}{\Gamma(n \alpha+1)}+J_{t}^{(n+1) \alpha} f(x, t)\right]+\cdots
\end{aligned}
$$

\section{HPTM solutions of non-linear space-time fractional reaction-diffusion equation}

Now, we consider the non-linear space-time fractional reaction-diffusion equation of the form:

$$
\begin{aligned}
& D_{t}^{\alpha} u(x, t)=b D_{x}^{\beta} u(x, t)+f(u(x, t))+g(x, t), 0<x<L, t>0,0<\alpha \leq 1,1<\beta \leq 2, \\
& u(x, 0)=p(x) .
\end{aligned}
$$

Taking the Laplace transform on both sides of Eq. (19) and using (20), we get

$$
L[u(x, t)]=\frac{p(x)}{s}+\frac{b}{s^{\alpha}} L\left[D_{x}^{\beta} u(x, t)\right]+\frac{1}{s^{\alpha}} L[f(u(x, t))]+\frac{1}{s^{\alpha}} L[g(x, t)] .
$$

Applying the inverse Laplace transform on both sides of Eq. (21), we get

$$
u(x, t)=p(x)+L^{-1}\left[\frac{b}{s^{\alpha}} L\left[D_{x}^{\beta} u(x, t)\right]+\frac{1}{s^{\alpha}} L[f(u(x, t))]\right]+J_{t}^{\alpha} g(x, t) .
$$

Now, we apply the homotopy perturbation method

$u(x, t)=\sum_{n=0}^{\infty} p^{n} u_{n}(x, t)$

and the nonlinear term can be decomposed as 


$$
f(u(x, t))=\sum_{n=0}^{\infty} p^{n} H_{n}(u)
$$

for some He's polynomials $H_{n}(u)[11,28]$ that are given by

$$
H_{n}\left(u_{0}, u_{1}, \ldots, u_{n}\right)=\frac{1}{n !} \frac{\partial^{n}}{\partial p^{n}}\left[f\left(\sum_{i=0}^{\infty} p^{i} u_{i}\right)\right]_{p=0}, \mathrm{n}=0,1,2,3 \ldots
$$

Substituting Eqs. (23) and (24) in Eq. (22), we get

$$
\begin{aligned}
& \sum_{n=0}^{\infty} p^{n} u_{n}(x, t)= \\
& \quad p(x)+p\left(L^{-1}\left[\frac{b}{s^{\alpha}} L\left[D_{x}^{\beta} \sum_{n=0}^{\infty} p^{n} u_{n}(x, t)\right]+\frac{1}{s^{\alpha}} L\left[\sum_{n=0}^{\infty} p^{n} H_{n}(u)\right]\right]+J_{t}^{\alpha} g(x, t) .\right.
\end{aligned}
$$

Comparing the coefficients of the like terms of $\mathrm{p}$, we have

$$
\begin{aligned}
p^{0}: u_{0}(x, t) & =p(x)+J_{t}^{\alpha} g(x, t), \\
p^{1}: u_{1}(x, t) & =L^{-1}\left[\frac{b}{s^{\alpha}} L\left[D_{x}^{\beta} u_{0}(x, t)\right]+\frac{1}{s^{\alpha}} L\left[H_{0}\right]\right] \\
= & J_{t}^{\alpha}\left[b D_{x}^{\beta} u_{0}(x, t)\right]+J_{t}^{\alpha}\left[H_{0}\right] .
\end{aligned}
$$

Proceeding in a similar manner, we get

$$
\begin{aligned}
& p^{2}: u_{2}(x, t)=J_{t}^{\alpha}\left[b D_{x}^{\beta} u_{1}(x, t)\right]+J_{t}^{\alpha}\left[H_{1}\right], \\
& p^{3}: u_{3}(x, t)=J_{t}^{\alpha}\left[b D_{x}^{\beta} u_{2}(x, t)\right]+J_{t}^{\alpha}\left[H_{2}\right], \\
& \vdots \\
& p^{n+1}: u_{n+1}(x, t)=J_{t}^{\alpha}\left[b D_{x}^{\beta} u_{n}(x, t)\right]+J_{t}^{\alpha}\left[H_{n}\right],
\end{aligned}
$$

and so on; in this manner, the rest of the components of the homotopy perturbation series can be obtained. Therefore, the solution in series form is given by

$$
\begin{aligned}
u(x, t) & =p(x)+J_{t}^{\alpha} g(x, t)+J_{t}^{\alpha}\left[b D_{x}^{\beta} u_{0}(x, t)\right]+J_{t}^{\alpha}\left[H_{0}\right]+ \\
& +J_{t}^{\alpha}\left[b D_{x}^{\beta} u_{1}(x, t)\right]+J_{t}^{\alpha}\left[H_{1}\right]+\ldots+J_{t}^{\alpha}\left[b D_{x}^{\beta} u_{n}(x, t)\right]+J_{t}^{\alpha}\left[H_{n}\right]+\cdots .
\end{aligned}
$$

\section{Numerical examples}

\section{Example 1}

Consider the following linear space-time fractional reaction-diffusion equation with boundary and initial conditions [46]:

$$
\begin{aligned}
& \frac{\partial^{\alpha} u(x, t)}{\partial t^{\alpha}}=b(x) \frac{\partial^{\beta} u(x, t)}{\partial x^{\beta}}-c(x) u(x, t)+f(x, t), 0 \leq x \leq 1,0 \leq t \leq 1, \\
& u(0, t)=u_{x}(0, t)=0, \\
& u(x, 0)=p(x)=x^{2}-x^{3},
\end{aligned}
$$

where the source function

$$
f(x, t)=3\left(4 t^{2}+1\right) x^{3}+\frac{32}{3 \sqrt{\pi}} t^{1.5}\left(x^{2}-x^{3}\right)
$$

the coefficients of the diffusion and reaction terms are $b(x)=\Gamma(1.2) x^{1.8}, c(x)=2$. If $\alpha=0.5, \beta=1.8$, the exact solution of this problem is $\left(4 t^{2}+1\right)\left(x^{2}-x^{3}\right)$, which can be verified by direct fractional differentiation of the given solution, and substituting in the fractional differential equation. The 
initial and boundary conditions are clearly satisfied. If $\alpha=0.5, \beta=1.8$, according to homotopy perturbation transform procedures Eqs. (9)-(18), we can successively obtain

$u_{0}(x, t)=\left(4 t^{2}+1\right)\left(x^{2}-x^{3}\right)+6 x^{3}\left(\frac{32 t^{2.5}}{15 \sqrt{\pi}}+\frac{t^{0.5}}{\sqrt{\pi}}\right)$

$u_{1}(x, t)=-3 x^{3}\left(\frac{64 t^{2.5}}{15 \sqrt{\pi}}+\frac{2 t^{0.5}}{\sqrt{\pi}}-4 t^{3}-3 t\right)$

$u_{2}(x, t)=-9 x^{3}\left(\frac{4 t^{3}}{3}-\frac{128 t^{3.5}}{15 \sqrt{\pi}}+t-\frac{2 t^{1.5}}{\sqrt{\pi}}\right)$

$u_{3}(x, t)=-27 x^{3}\left(\frac{4 t^{1.5}}{3 \sqrt{\pi}}-\frac{128 t^{3.5}}{105 \sqrt{\pi}}-t^{4}-\frac{3 t^{2}}{2}\right)$

and so on; in this manner, the rest of the components of the homotopy perturbation series can be obtained. Thus, we have the solution in series form is given by

$u(x, t)=u_{0}(x, t)+u_{1}(x, t)+u_{2}(x, t)+u_{3}(x, t)+\cdots$.

When $\alpha=0.5, \beta=1.8$, Figs. (1-2-3-4-5) show the different approximate solutions obtained by applying the HPTM and the exact solutions of space-time fractional reaction-diffusion equation.

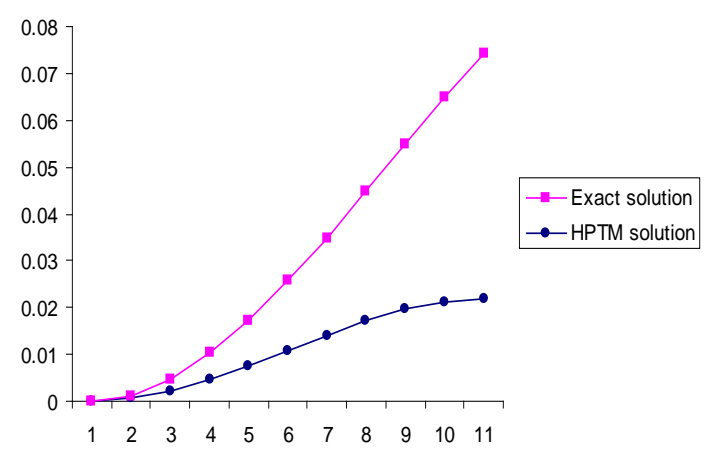

Fig. 1 Comparison of the exact solution and approximate solution at time $t=0.4$ for $\alpha=0.5$ and $\beta=$ 1.8 .

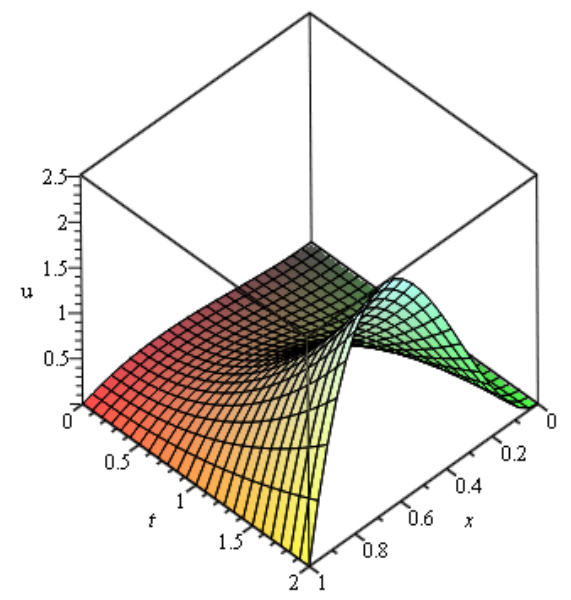

Fig. 2 Exact solution graph of Example 1 at $\alpha=0.5$ and $\beta=1.8$ at $\mathrm{t}=0$ to $\mathrm{t}=2$. 


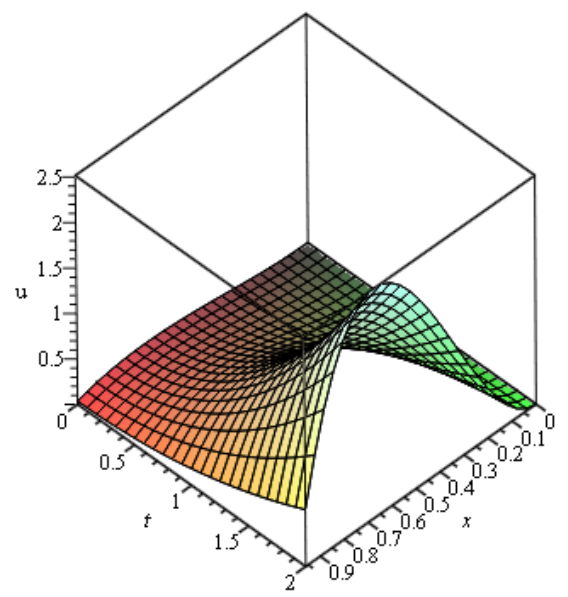

Fig. 3 Approximate solution graph of Example 1 at $\alpha=0.5$ and $\beta=1.8$ up to fourth approximation at $t=0$ to $t=5$ by HPTM.

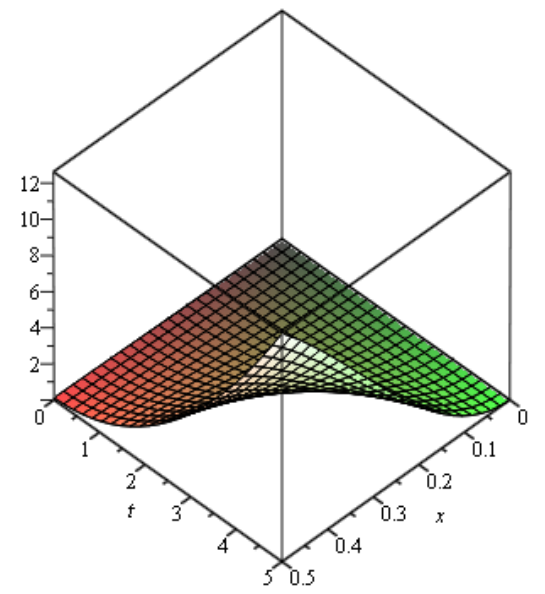

Fig. 4 Exact solution graph of Example 1 at $\alpha=0.5$ and $\beta=1.8$ at $t=0$ to $t=5$.

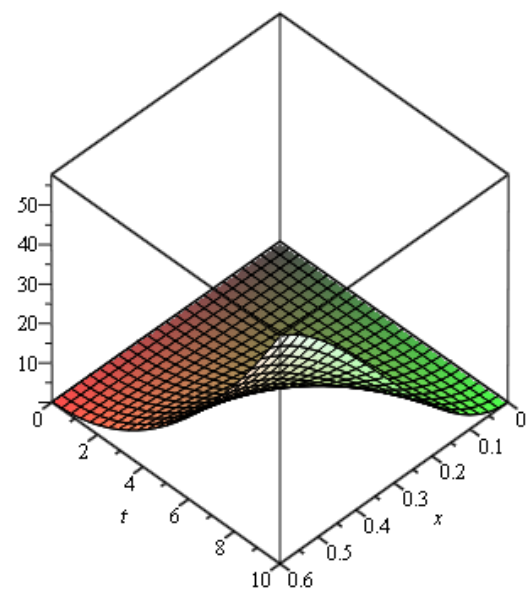

Fig. 5 Approximate solution graph of Example 1 at $\alpha=0.5$ and $\beta=1.8$ up to fourth approximation at $t=0$ to $t=10$ by HPTM.

\section{Example 2}

Consider the following non-linear space-time fractional reaction-diffusion equation with boundary and initial conditions [46]: 
$\frac{\partial^{\alpha} u(x, t)}{\partial t^{\alpha}}=b \frac{\partial^{\beta} u(x, t)}{\partial x^{\beta}}+f(u(x, t))+g(x, t), 0 \leq x \leq 1, t>0$,

$u(x, 0)=0,0 \leq x \leq 1$,

where the non-linear reaction terms in Fisher's growth equation:

$f(u(x, t))=0.25 u(x, t)(1-u(x, t))$

and

$g(x, t)=-0.0104649 t^{0.9}+0.00961766 x^{1.1}-0.0025 t^{0.9} x^{1.1}+0.000025 t^{1.8} x^{2.2}$.

If $\alpha=0.9, \beta=1.1$, the exact solution of this problem is $0.01 x^{1.1} t^{0.9}$, which can be verified by direct fractional differentiation of the given solution, and substituting in fractional differential equation. The initial and boundary conditions are clearly satisfied. Using Eqs. (22), (24) and (25), the first few components of He's polynomials $H_{n}$ that represent the non-linear term $0.25 \mathrm{u}(\mathrm{x}, \mathrm{t})(1-\mathrm{u}(\mathrm{x}, \mathrm{t}))$ are obtained as

$$
\begin{aligned}
& H_{0}=0.5 u_{0}\left(1-u_{0}\right), \\
& H_{1}=u_{1}\left(0.25-0.5 u_{0}\right), \\
& H_{2}=u_{2}\left(0.25-0.5 u_{0}\right)+\frac{u_{1}^{2}}{2}(0.5), \\
& H_{3}=u_{3}\left(0.25-0.5 u_{0}\right)+u_{1} u_{2}(0.5),
\end{aligned}
$$

If $\alpha=0.9, \beta=1.1$, using (37), according to homotopy perturbation transform procedures Eqs. (21)(32) we now successively obtain

$$
\begin{aligned}
u_{0}(x, t)= & 0.01 x^{1.1} t^{0.9}-0.0143419 x^{1.1} t^{1.8}-0.0600346 t^{1.8}+0.0000100493 x^{2.2} t^{2.7}, \\
u_{1}(x, t)= & 0.00600346 t^{1.8}-0.00120662 t^{2.7}-2.30348 \times 10^{-6} t^{4.5}+\left(0.00143419 t^{1.8}-\right. \\
& \left.0.000144127 t^{2.7}+0.0000166107 t^{3.6}-1.10058 \times 10^{-6} t^{4.5}\right) x^{1.1}+ \\
& \left(-0.0000100493 t^{2.7}+3.01807 \times 10^{-6} t^{3.6}-1.31461 \times 10^{-7} t^{4.5}+\right. \\
& \left.6.5514 \times 10^{-9} t^{5.4}\right) x^{2.2}+\left(-1.28454 \times 10^{-8} t^{4.5}+1.56623 \times 10^{-9} t^{5.4}\right) x^{3.3}+ \\
& \left(-4.78235 \times 10^{-12} t^{6.3}\right) x^{4.4}, \\
u_{2}(x, t)= & 0.00120662 t^{2.7}-0.000141028 t^{3.6}+9.05084 \times 10^{-6} t^{4.5}-1.16267 \times 10^{-6} t^{5.4} \\
& -1.1625 \times 10^{-9} t^{7.2}+\left(0.000144127 t^{2.7}-0.000027841 t^{3.6}+\right. \\
& 6.59228 \times 10^{-6} t^{4.5}-4.08064 \times 10^{-7} t^{5.4}+1.45028 \times 10^{-8} t^{6.3}- \\
& \left.8.33145 \times 10^{-10} t^{7.2}\right) x^{1.1}+\left(-3.01807 \times 10^{-6} t^{3.6}+6.40041 \times 10^{-7} t^{4.5}-\right. \\
& 7.0968 \times 10^{-8} t^{5.4}+7.55741 \times 10^{-9} t^{6.3}-1.9903410^{-10} t^{7.2}+ \\
& \left.4.72952 \times 10^{-12} t^{8.1}\right) x^{2.2}+\left(1.28454 \times 10^{-8} t^{4.5}-7.11015 \times 10^{-9} t^{5.4}+\right. \\
& \left.7.45807 \times 10^{-10} t^{6.3}-4.59253 \times 10^{-11} t^{7.2}+2.25971 \times 10^{-12} t^{8.1}\right) x^{3.3} \\
& +\left(2.17306 \times 10^{-11} t^{6.3}-5.61593 \times 10^{-12} t^{7.2}+2.69917 \times 10^{-13} t^{8.1}-\right. \\
& \left.-6.51357 \times 10^{-15} t^{9.0}\right) x^{4.4}+\left(1.33856 \times 10^{-14} t^{8.1}-\right. \\
& \left.1.55606 \times 10^{-15} t^{9.0}\right) x^{5.5}+\left(3.03862 \times 10^{-18} t^{9.9}\right) x^{6.6},
\end{aligned}
$$

and so on; in this manner, the rest of components of the homotopy perturbation series can be obtained. Thus, we have the solution in series form is given by

$u(x, t)=u_{0}(x, t)+u_{1}(x, t)+u_{2}(x, t)+u_{3}(x, t)+\cdots$. 
When $\alpha=0.9, \beta=1.1$, Figs. (6-7-8) show the different approximate solutions obtained by applying the HPTM and the exact solutions of space-time fractional reaction-diffusion equation. From Fig. 6 , it can be seen that the approximate solution is in excellent agreement with the exact solution.

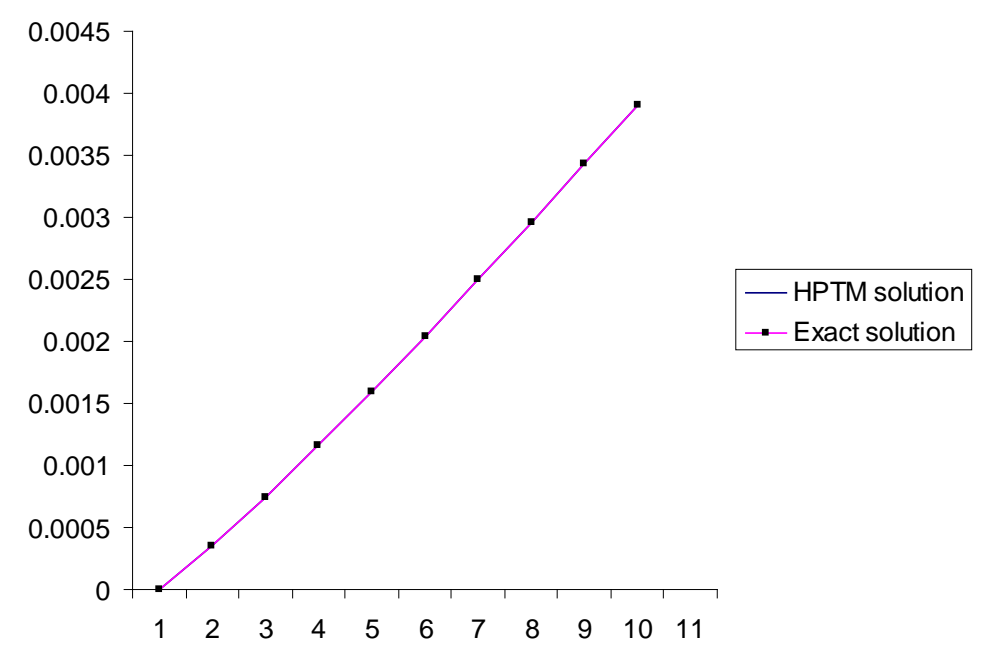

Fig. 6 Comparison of the exact solution and approximate solution at time $t=0.4$ for $\alpha=0.9$ and $\beta=$ 1.1.

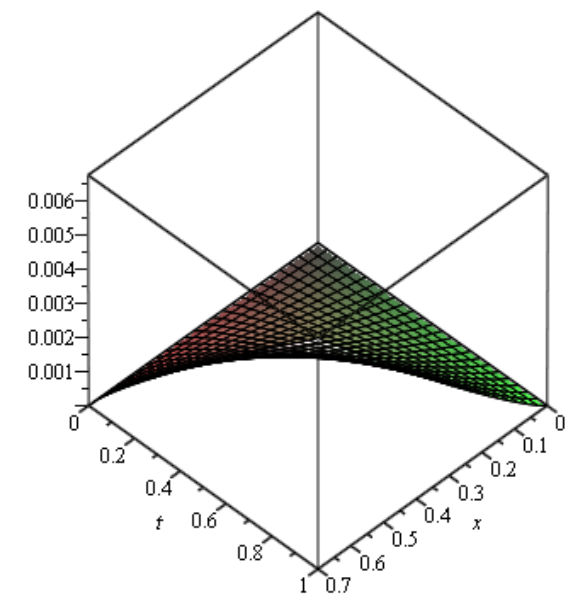

Fig. 7 Exact solution graph of Example 2 at $\alpha=0.9$ and $\beta=1.8$ at $t=0$ to $t=1$.

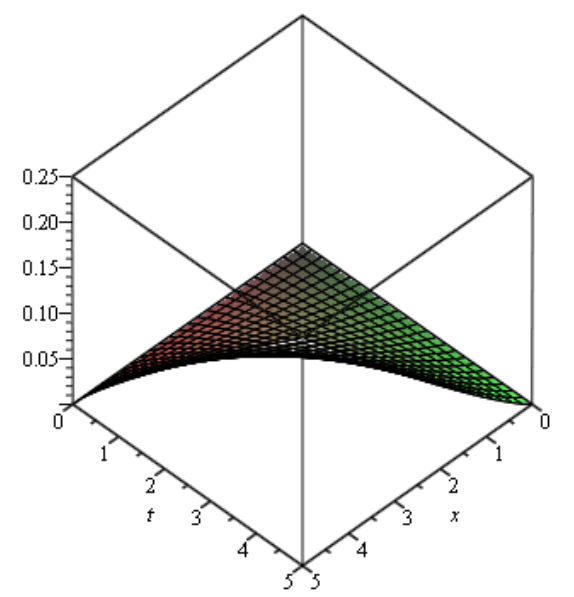

Fig. 8 Approximate solution graph of Example 2 at $\alpha=0.9$ and $\beta=1.1$ at $t=0$ to $t=5$ up to fourth approximation by HPTM. 


\section{Conclusions}

In this paper, we have used HPTM for solving the linear and non-linear space-time fractional diffusion equations. The HPTM is clearly a very efficient technique for finding the solutions of the proposed equations. It is interesting to note that HPTM is an elegant combination of the Laplace transformation and the homotopy perturbation method. The mathematical technique employed in the present article is significant in studying some other problems of engineering and physics.

\section{References.}

[1] B. Baeumer, M. Kovacs and M. Meerschaert, Numerical solution for fractional reaction- diffusion equation, Computers and Mathematics with Applications, 55(2008), 2212-2226.

[2] M. Bar, N. Gottschalk, M. Eiswirth and G. Ertl, Spiral waves in a surface reaction: model calculations, Journal of Chemical Physics, 100 (1994), 1202-1214.

[3] D.A. Benson, S. Wheatcraft and M.M. Meerschaert, Application of a fractional advection dispersion equation, Water Resource Research, 36 (2000), 1403-1412.

[4] M.A. Burke, P.K. Miani and J.D. Murray, Suicide substrate reaction-diffusion equations varying the source, IMA Journal Mathematical Applications in Medicine and Biology, 10 (1993), 97-114.

[5] M. Caputo, Elasticitá e Dissipazione, Zani-chelli, Bologna, 1969.

[6] L. Cong-Xin, C. Xiao-Fang, W. Peng-Ye and W. Wei-Chi, Effects of CRAC channel on spatiotemporal $\mathrm{Ca}^{+2}$ patterns in T cells, Chinese Physics Letters, 27 (2010), 028701.

[7] S. Das, Solution of Fractional Vibration Equation by the Variational Iteration Method and Modified Decomposition Method, International Journal of Nonlinear Science and Numerical Simulation, 9 (2008), 361-366.

[8] R. Erban and S.J. Chapman, Stochastic modeling of reaction diffusion processes: algorithms for bimolecular reactions, Physical Biology, 6 (2009), 046001.

[9] Z.Z. Ganji, D.D Ganji and H. Jafari, Application of the homotopy perturbation Method to coupled system of partial differential equations with time fractional derivatives, Topological Methods in Nonlinear Analysis, 31 (2008), 341-348.

[10] A. Ghorbani and J. Saberi-Nadjafi, He's homotopy perturbation method for calculating adomain polynomials, International Journal of Nonlinear Sciences and Numerical Simulation, 8 (2007), 229232.

[11] A. Ghorbani, Beyond Adomain's polynomials: He's polynomials, Chaos Solitons, Fractals, 39 (2009), 1486-1492.

[12] R. Gorenflo, F. Mainardi, E. Scalas and M. Raberto, Fractional calculus and continuous time finance. III: The diffusion limit. Mathematical finance. Konstanz, 2000. Trends Math Birkhuser Basel, (2001), 171-180. 
[13] V. Grafiychuk, B. Datsko and S.V. Meleshko, Mathematical modeling of pattern formation in sub- and superdiffusive reaction diffusion systems, arxiv: nlin.AO/06110005 v3, (2006).

[14] V. Grafiychuk, B. Datsko and S.V. Meleshko, Nonlinear oscillations and stability domains in fractional reaction-diffusion systems, arxiv: nlin.PS/0702013 v1, (2007).

[15] M.J. Grimson and G.C. Barker, A continuum model for the growth of bacterial colonies on a surface, Journal of Physics A: Mathematical General, 26 (1993), 5645-5654.

[16] J.H. He, Approximate analytical solution for seepage flow with fractional derivatives in porous media, Comput. Methods Appl. Mech. Eng., 167 (1998), 57-68.

[17] J.H. He, Homotopy perturbation technique, Computer Methods in Applied Mechanics and Engineering, 178 (1999), 257-262.

[18] B.I. Henry and S.L. Wearne, Fractional reaction-diffusion. Physica A, 276 (2000), 448-455.

[19] M. Inc, He's homotopy perturbation method for Solving Korteweg-de Veries Burgers Equation with Initial Condition, Numerical Methods for Partial Differential Equations, 26(5) (2009), 12241235.

[20] H. Jafari and S. Momani, Solving fractional diffusion and wave equations by modified homotopy perturbation method, Physics Letters A, 370 (2007), 388-396.

[21] N.A. Khan, N. Khan, A. Ara and M. Jamil, Approximate analytical solutions of fractional reactiondiffusion equations, Journal of King Saud University (Science), 24(2) (2012), 111-118.

[22] Y. Khan and Q. Wu, Homotopy perturbation transform method for nonlinear equations using He's polynomials, Computer and Mathematics with Applications, 61(6) (2011), 1963-1967.

[23] M. Khan, M.A. Gondal and S. Kumar, A new analytical approach to solve exponential stretching sheet problem in fluid mechanics by variational iterative Padé method, The Journal of Mathematics and Computer Science, 3 (2) (2011), 135-144.

[24] A.A. Kilbas, H.M. Srivastava and J.J. Trujillo, Theory and applications of Fractional Differential Equations, Elsevier, Amsterdam, 2006.

[25] F. Mainardi, M. Raberto, R.Gorenflo and R. Scalas, Fractional calculus and continuous-time finance. II: The waiting-time distribution, Physica A, 287(2000), 468-481.

[26] M. Matinfar and M. Ghanbari, Solution of systems of integral-differential equations by variational iteration method, The Journal of Mathematics and Computer Science, 1(1) (2010), 4657.

[27] K.S. Miller and B. Ross, An introduction to the Fractional Calculus and Fractional Differential Equations, Wiley, New York, 1993.

[28] S.T. Mohyud-Din, M.A. Noor and K.I. Noor, Travelling wave solutions of seventh- order generalized KdV equations using He's polynomials, Int. J. Nonlin. Sci. Num. Sim., 10 (1) (2009), 227-233.

[29] S. Momani and Z. Odibat, Homotopy perturbation method for nonlinear partial differential equations of fractional order, Physics Letters A, 365 (2007), 345-350. 
[30] S. Momani, Z. Odibat and I. Hasshim, Algorithms for non-linear fractional partial differential equations: A selection of numerical methods, Topological Methods in Nonlinear Analysis, 31 (2008), 211-226.

[31] S.C. Muller, T. Plesser and B. Hess, Two-dimensional spectrophotometry of spiral wave propagation in the Belousov-Zhabotinskii reaction. I: Experiments and digital data representation, Physica D, 24 (1987), 71-78.

[32] J.D. Murray, Lectures on Non-Linear Differential Equation Models in Biology. Clarenden, Oxford, (1977).

[33] Z. Odibat and S. Momani, Application of variational iteration method to nonlinear differential equations of fractional order, International Journal of Nonlinear Science and Numerical Simulation, 7 (2006), 27-34.

[34] Z. Odibat and S. Momani, Application of variational iteration and homotopy perturbation methods to fractional evolution equations, Topological Methods in Nonlinear Analysis, 31 (2008) $227-234$.

[35] M.I.A. Othman, A.M.S. Mahdy and R.M. Farouk, Numerical solution of $12^{\text {th }}$ order boundary value problems by using homotopy perturbation method, The Journal of Mathematics and Computer Science, 1(1) (2010), 14-27.

[36] M.I.A. Othman and A.M.S. Mahdy, Differential transformation method and variation iteration method for Cauchy reaction-diffusion problems, The Journal of Mathematics and Computer Science, 1(2) (2010), 61-75.

[37] M. Raberto, E. Scalas and F. Mainardi, Waiting-times and returns in high frequency financial data: an empirical study, Physica A, 314 (2002), 749-755.

[38] K. Seki, M. Wojcik and Tachiya, Fractional reaction- diffusion equation, Journal of Chemical Physics, 119(2003), 2165-2174.

[39] B.M. Slepchenko, J.C. Schaff and Y.S. Choi, Numerical approach to fast reactions in reactiondiffusion systems: application to buffered calcium waves in bistable models, Journal of Computational Physics, 162 (2000), 186-189.

[40] C. Vidal and A. Pascault, Non-equilibrium Dynamics in Chemical Systems, Wiley, New York, (1986).

[41] A.T. Winfree, Spiral waves of chemical activity, Science, 175, (1972), 634-642.

[42] A. Yildirim, Solution of BVPs for Fourth- Order Integro-Differential Equations by using Homotopy Perturbation Method, Computers \& Mathematics with Applications, 56 (2008), 31753180.

[43] A. Yildirim, An Algorithm for Solving the Fractional Nonlinear Schröndinger Equation by Means of the Homotopy Perturbation Method, International Journal of Nonlinear Science and Numerical Simulation, 10(2009), 445-451. 
J. Singh, D. Kumar, Sushila, S. Gupta / TJ MCS Vol .5 No.1 (2012) 40-52

[44] A. Yildirim, He's homotopy perturbation method for solving the space- and time- fractional telegraph equations, International Journal of Computer Mathematics, 87(13) (2010), 2998-3006.

[45] A. Yildirim and S.A. Sezer, Analytical solution of linear and non-linear space-time fractional reaction-diffusion equations, International Journal of Chemical Reactor Engineering, 8(2010), 1-21.

[46] Q. Yu, F. Liu, V. Anh and I. Turner, Solving linear and non-linear Space-time fractional reactiondiffusion equations by the Adomian decomposition method, Int. J. Numer. Meth. Engg., 74(2008), 138-158. 